\title{
Diagnosing postpartum depression in a mother of developmentally delayed infant: a case report
}

\author{
Genco Usta1, Yaşam Kemal Akpak ${ }^{2} *$, Onat Yılmaz ${ }^{3}$ \\ ${ }^{1}$ Boylam Psychiatry Hospital, Department of Child and Adolescence Psychiatry, Ankara, Turkey \\ ${ }^{2}$ Ankara Mevki Military Hospital, Department of Obstetrics and Gynecology, Ankara, Turkey \\ ${ }^{3}$ Kasımpaşa Military Hospital, Department of Psychiatry, Istanbul, Turkey
}

Received: 09 September 2015

Accepted: 21 October 2015

\section{*Correspondence:}

Dr. Yasam Kemal Akpak,

E-mail: yasamaster@gmail.com

Copyright: $\odot$ the author(s), publisher and licensee Medip Academy. This is an open-access article distributed under the terms of the Creative Commons Attribution Non-Commercial License, which permits unrestricted non-commercial use, distribution, and reproduction in any medium, provided the original work is properly cited.

\begin{abstract}
Postpartum depression is the most common clinical entity of childbirth. Although there are conflicting results about the influence of postpartum depression on the children, the consequences of the illness might extend to preschool age or further. Because physicians encounter mothers at the same time with the babies in the examination room, it might be of particular importance to have the knowledge of symptoms of maternal depression and pay attention to the mental and physical well-being of both the mother and the baby. In this article we would like to present an 18 month old child who presented with psychomotor retardation with a mother diagnosed as postpartum depression.
\end{abstract}

Keywords: Antidepressants, Child development, Postpartum depression, Pregnancy, Psychotherapy

\section{INTRODUCTION}

Postpartum depression is a depressive disorder occurring in mothers during the postpartum period with an estimated prevalence of between $10 \%$ and $15 \%{ }^{1}$ It is characterized by various substantial depressive symptoms like anhedonia, uncontrollable mood swings, loss of energy and motivation, a fear of being alone, sleep disturbances and withdrawal and isolation from friends and family. ${ }^{2}$ Women are susceptible to mental disorders during reproductive years and risk factors for developing postpartum depression include having a history of mood disorders, depression and anxiety symptoms during pregnancy, stressful life events and a family history of psychiatric disorders. ${ }^{3}$ Postpartum depression might have negative consequences on mother's quality of life, as well as the other family members and the baby. In this article, we will present a clinical case of a baby that highlights common clinical challenges in diagnosing postpartum depression in the mother and try to raise the awareness of physicians for postpartum depression in mothers when examining the baby.

\section{CASE REPORT}

An 18 month old male child referred to our outpatient clinic by his parents for speech delay, not responding to his name, low interest to environment, poor eye contact and loss of appetite. He was born by spontaneous vaginal delivery at term and the birth weight was 3800 grams. Hearing assessment at three days of birth was in normal limits. He was breast-fed for about 11 months. His physical examination was normal, had no dysmorphic features or abnormal neurological signs. Physical examination showed general developmental retardation corresponding to fine motor developmental skills of a 12 month level and he scored on language skills at the level of a nine-month. Complete blood count, biochemical analysis, metabolic parameters (e.g. amino acids, lactate in blood and amino acids in urine) and laboratory tests for 
possible viruses and other parasites were normal. Chromosomal evaluation revealed any genetic syndrome and brain magnetic resonance imaging (MRI) scans and electroencephalogram (EEG) was normal. Although some autistic behaviors were observed in his psychiatric evaluation, clinical picture of the patient was more concordant with developmental delay due to environmental deprivation. Mother's psychiatric evaluation revealed irritability and depressed mood starting within 3th week after birth, and concentration problems, frequent crying episodes, sleep disturbances and headache attacks twice in a week for the last 2 months. Although mother had a healthy pregnancy period, she significantly began to have difficulty in controlling her emotions; she often felt tired, hopeless and helpless and lost her interest in daily activities (e. g. feeding the baby, cooking) after birth. Past medical history of mother did not involve acute stressors, and alcohol or any illegal drug abuse. Mother was diagnosed with postpartum depression according to DSM-IV TR criteria and had score of 32 in Hamilton Psychiatric Rating Scale for Depression (HAM-D-21). ${ }^{4}$ After a 6 month-long treatment with sertraline $50 \mathrm{mg}$ per day and structured cognitive behavioral therapy sessions, Hamilton Psychiatric Rating Scale for Depression (HAMD-21) score regressed to 8. Parents were also included in a training program aiming to teach parents how to communicate with child and respond with appropriate behaviors to child's emotional and psychological needs. Developmental screening test of the baby after 3 months of treatment and 6 months after treatment, resulted in fine motor development of a 15, 26 month-level, and scored on language development at the level of 16 and 22 months, respectively.

\section{DISCUSSION}

Depression is a severe mental disorder having multiple adverse effects on the patients and the society. Although postpartum depression affects $10 \%-15 \%$ of women after delivery, less than half of the cases are diagnosed in clinical practice. ${ }^{3}$ Moreover, recent evidence indicates that only limited number $(18 \%)$ of women seek treatment for their depressive complaints during pregnancy and postpartum period. ${ }^{5}$

There is good evidence that postpartum depression is associated with disturbed maternal-new-born attachment, disturbed mother-infant interaction, cognitive and language development delays in baby, negative parenting practices, troubles in breastfeeding, ${ }^{6}$ increased vulnerability of the mother to poor quality of life, depression among fathers. ${ }^{7}$ It has been noted that the impact of postpartum depression has various outcomes based on the duration, severity and timing of depression, and aggravating risk factors like low social support and financial stress. Because depressed mothers are affectively flat and unresponsive in common, infants develop withdrawal, passivity and self-regulatory behaviours in response. Studies claimed that infants of depressed mothers have more internalizing and externalizing problems, show patterns of arousal, lower cognitive performance and diminished attention. ${ }^{8}$

Postpartum depression might affect the offspring beyond infanthood and its consequences might extend to preschool age, school age and even adolescence. ${ }^{8}$ Children and adolescents with postpartum depressed mothers might have difficulties in mathematic reasoning and lower cognitive performance, attention deficit/hyperactivity disorder and lower IQ scores, psychopathologies such as affective disorders (depression), anxiety disorders, substance abuse, phobias, panic disorders, conduct disorders and alcohol dependence are detected in higher rates. ${ }^{8}$

Taking all the available data into account, it is extremely important to detect and treat all women who experience symptoms of depression after birth. Screening the mother and the baby improves case identification. Once depression is suspected, the clinician can guide both the mother and the baby for appropriate treatment options. Treatment alternatives for portpartum depression include pharmacotherapy, psychotherapy and combination of both. Treatment choice is driven by the severity of symptoms, patient preferences and antidepressant therapy should be considered with the patient in the context of risk-benefit assessment. Cognitive-behavioral therapy and interpersonal therapy are reported to be effective in treating postpartum depression. Transcranial magnetic stimulation for selected cases and electroconvulsive therapy for severe cases can be considered. ${ }^{6}$

\section{Funding: No funding sources \\ Conflict of interest: None declared \\ Ethical approval: Not Required}

\section{REFERENCES}

1. Couto TC, Brancaglion MYM, Alvim-Soares A, Moreira L, Garcia FD, Nicolato R, et al. Postpartum depression: A systematic review of the genetics involved. World J Psychiatr. 2015;5:103-11.

2. American Psychiatric Association. Diagnostic and statistical manual of mental disorders: DSM-IV-TR. 4th ed., Text Revision: Washington, DC; 2000.

3. Gjerdingen DK, Yawn BP. Postpartum depression screening: importance, methods, barriers, and recommendations for practice. J Am Board Fam Med. 2007;20:280-8.

4. Hamilton M. A Rating scale for depression. J Neurol Neurosurg Psychiat. 1960;23:56-62.

5. Marcus SM. Depression during pregnancy: rates, risks and consequences--Motherisk Update 2008. Can J Clin Pharmacol. 2009; 16:15-22.

6. Bobo VW, Yawn BP. Concise review for physicians and other clinicians: Postpartum depression. Mayo Clin Proc. 2014;89:835-44. 
7. Rai S, Pathak A, Sharma I. Postpartum psychiatric disorders: Early diagnosis and management. Indian J Psychiatry. 2015;57:216-21.

8. Sohr-Preston SL, Scaramella LV. Implications of timing of maternal depressive symptoms for early cognitive and language development. Clin Child Fam Psychol Rev. 2006;9:65-83.

Cite this article as: Usta G, Akpak YK, Y1lmaz O. Diagnosing Postpartum Depression in a mother of developmentally delayed infant: a case report. Int $\mathbf{J}$ Reprod Contracept Obstet Gynecol 2015;4:2056-8. 